\title{
Role of Cocontraction in Arm Movement Accuracy
}

\author{
Paul L. Gribble, ${ }^{1,2,3}$ Lucy I. Mullin, ${ }^{1,2}$ Nicholas Cothros, ${ }^{1,3}$ and Andrew Mattar ${ }^{1,2}$ \\ ${ }^{1}$ Department of Psychology, ${ }^{2}$ Department of Physiology and Pharmacology, ${ }^{3}$ Graduate Program in Neuroscience, The University of \\ Western Ontario, London, Ontario N6A 5C2, Canada
}

Submitted 11 November 2002; accepted in final form 18 January 2003

\begin{abstract}
Gribble, Paul L., Lucy I. Mullin, Nicholas Cothros, and Andrew Mattar. Role of cocontraction in arm movement accuracy. J Neurophysiol 89: 2396-2405, 2003. First published January 22, 2003; 10.1152/jn.01020.2002. Cocontraction (the simultaneous activation of antagonist muscles around a joint) provides the nervous system with a way to adapt the mechanical properties of the limb to changing task requirements-both in statics and during movement. However, relatively little is known about the conditions under which the motor system modulates limb impedance through cocontraction. The goal of this study was to test for a possible relationship between cocontraction and movement accuracy in multi-joint limb movements. The electromyographic activity of seven single- and double-joint shoulder and elbow muscles was recorded using surface electrodes while subjects performed a pointing task in a horizontal plane to targets that varied randomly in size. Movement speed was controlled by providing subjects with feedback on a trial-to-trial basis. Measures of cocontraction were estimated both during movement and during a $200-\mathrm{ms}$ window immediately following movement end. We observed an inverse relationship between target size and cocontraction: as target size was reduced, cocontraction activity increased. In addition, trajectory variability decreased and endpoint accuracy improved. This suggests that, although energetically expensive, cocontraction may be a strategy used by the motor system to facilitate multi-joint arm movement accuracy. We also observed a general trend for cocontraction levels to decrease over time, supporting the idea that cocontraction and associated limb stiffness are reduced over the course of practice.
\end{abstract}

\section{N T R O D U C T I O N}

By simultaneously activating antagonist muscles around a joint, the CNS can adapt the mechanical properties of the limb in response to task requirements-both in holding posture and during limb movements. Changes in muscle cocontraction affect joint impedance, which provides mechanical stability in the presence of external perturbations and forces due to limb dynamics. Relatively little is known, however, about the conditions under which the CNS modulates limb impedance through cocontraction or how central commands related to cocontraction are coordinated with those related to movement production. A greater understanding of the behavioral determinants of cocontraction is needed to more fully characterize the rich repertoire of control strategies employed by the CNS when controlling the limbs. In addition, this information could be important for constraining computational models of movement control that postulate distinct central commands for cocontraction (Bhushan and Shadmehr 1999; Feldman and Levin

Address for reprint requests: P. L. Gribble, Dept. of Psychology, Univ. of Western Ontario, London, Ontario N6A 5C2, Canada (E-mail: pgribble@uwo.ca).
1995; Gribble and Ostry 2000; Gribble et al. 1998; Todorov 2000).

Behavioral studies of limb postural control have shown that subjects are able to modulate the coactivation of antagonist muscles around a joint to minimize the perturbing effects of external loads (De Serres and Milner 1991; Kearney and Hunter 1990; Latash 1992; Milner 2002; Milner and Cloutier 1998). Studies in the context of single- and multi-joint limb movements have shown that cocontraction increases with movement velocity and with the magnitude of perturbing forces due to limb dynamics (Gribble and Ostry 1998; Suzuki et al. 2001), and that cocontraction decreases gradually over the course of learning a novel motor task (Osu et al. 2002; Thoroughman and Shadmehr 1999). The goal of the present study is to assess the role of cocontraction in movement accuracy.

Constraints on accuracy have long been demonstrated to affect kinematic parameters of limb movement such as speed, which for rapid aiming movements, increases as target size increases (Fitts 1954; Fitts and Peterson 1964; Soechting 1984). Here we test the hypothesis that the CNS modulates cocontraction for arm movements in the context of different accuracy constraints. Increases in joint stiffness brought about by muscle cocontraction would have a beneficial effect on limb stability and hence movement accuracy by reducing the perturbing effects of joint interaction torques (Gribble and Ostry 1999; Koshland et al. 2000) and external forces (Gomi and Haggard 2001; Lacquaniti and Maioli 1989; Osu et al. 2002; Thoroughman and Shadmehr 1999).

We examined patterns of muscle activation using surface EMG for a multi-joint pointing task in which target size and location were varied. We show that trajectory variability decreased and endpoint accuracy improved as target size decreased, and that this improvement in accuracy was accompanied by an increase in cocontraction of antagonist muscles at the shoulder and elbow. These results suggest that despite the energetic cost of muscle coactivation, the CNS may use cocontraction as a strategy to facilitate limb movement accuracy.

\section{MET HOD S}

\section{Subjects}

16 subjects ( 8 females, 8 males) between the ages of 20 and 30 participated in the study. All subjects were right-handed with normal

\footnotetext{
The costs of publication of this article were defrayed in part by the payment of page charges. The article must therefore be hereby marked "advertisement" in accordance with 18 U.S.C. Section 1734 solely to indicate this fact.
} 
or corrected vision and reported having no history of neurological or musculo-skeletal disorders. All procedures were approved by the University of Western Ontario Research Ethics Board.

\section{Apparatus}

Subjects were seated in the dark in front of a glass tabletop with their right arm abducted at the shoulder and supported by custom made air-sleds in a horizontal plane containing the shoulder. In this configuration, the shoulder and elbow joints were free to rotate, providing subjects with two degrees of freedom of motion. The effect of the air-sleds, which were connected to a 40-psi compressed air source, was to support the arm against gravity and to reduce friction during movement. Medium-density Temper foam (Kees Goebel Medical) was used to provide a cushion between the arm and the air-sleds, and as a result the arm was suspended about $10 \mathrm{~cm}$ above the surface of the glass tabletop. One air-sled was placed under the upper arm and the other under the wrist. The index finger was extended and stabilized with a $2 \times 10 \mathrm{~cm}$ plastic splint that was placed under the wrist, extended to the end of the index finger, and secured with medical tape.

A computer controlled LCD projector displayed visual targets in a virtual plane in front of subjects. Targets were back-projected onto a screen suspended $20 \mathrm{~cm}$ above the hand and were reflected into view of subjects by a semi-silvered mirror positioned $10 \mathrm{~cm}$ below the screen. This resulted in the perception of virtual targets "floating" in the plane of the subject's hand. Two small lamps illuminated the area below the mirror, providing subjects full visual feedback of their arm during the experiment.

\section{Experimental task}

Subjects were asked to perform rapid pointing movements from a central start circle to targets which varied in size and location (see Fig. 1). At the beginning of each trial, subjects were instructed to point, using the tip of their extended index finger, to the start circle $(20 \mathrm{~mm}$ radius). The start circle was located $5 \mathrm{~cm}$ to the left and $35 \mathrm{~cm}$ in front of the shoulder. After maintaining this limb position for $1,500 \mathrm{~ms}$, the start circle was extinguished, and a target, either 5,30 , or $45 \mathrm{~mm}$ in radius, appeared in one of four different locations, $15 \mathrm{~cm}$ from the center of the start circle. Subjects were instructed to move to the target using one continuous motion, making no corrective movements during or after movement end, and to hold the final limb position until the

A

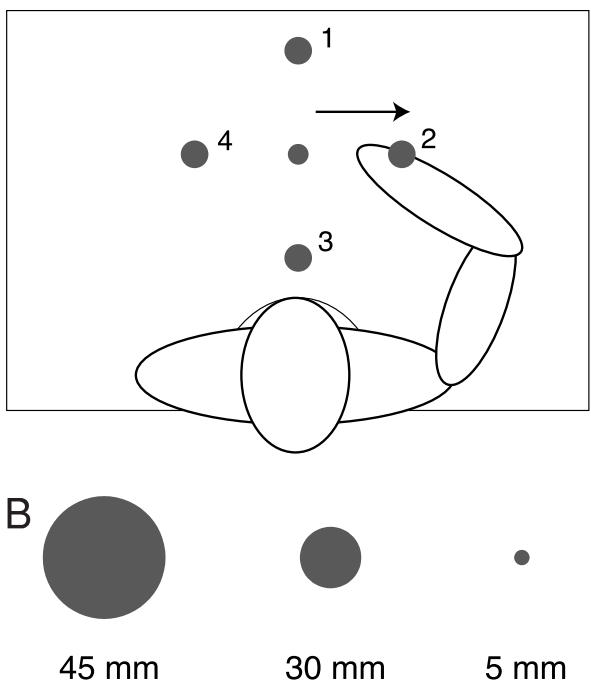

FIG. 1. Schematic showing target sizes and locations. Movements were executed in a horizontal plane containing the shoulder, which was abducted $90^{\circ}$. beginning of the subsequent trial. To successfully hit each target (which was indicated by the target changing color), subjects had to land within a "virtual" boundary around each target; virtual boundaries were 20,35 , and $50 \mathrm{~mm}$, for the 5,30 , and $45 \mathrm{~mm}$ targets, respectively. Trials in which subjects did not land within the virtual boundary were repeated.

To control for the possibility that any observed differences in EMG were due to variation in kinematic parameters such as speed, movement speed was controlled by providing subjects with feedback on a trial-to-trial basis about movement timing. Only movements with durations within a 60 -ms target window around the desired duration (475 ms) were recorded and included in subsequent analyses. If a movement was too slow or too fast, the screen briefly flashed green or red, respectively. If a movement was not achieved within the required time window, the trial was repeated. Typically after a brief practice session (5 or 10 movements) subjects were able to perform the task while maintaining the required timing constraints. Each subject completed a total of 240 movement trials $(20$ repetitions $\times 4$ target positions $\times 3$ target sizes). Target sizes and positions were fully randomized across trials for each subject. The number of repeated trials ranged across subjects from 5 to $15 \%$ of the total number of trials. There was no significant difference between the number of repeated trials for the 45 versus $30 \mathrm{~mm}$ targets (mean number of repeated trials was 7.0 and $6.7 \%$ for 45 and $30 \mathrm{~mm}$ targets, respectively; $P>0.05$ ) and only a slight (but statistically reliable) difference for the 45 and $30 \mathrm{~mm}$ targets compared with the 5-mm target (mean number of repeated trials was $8.7 \%$ for the 5 -mm target; $P<0.05$ in both cases).

\section{Signal recording}

The position of the limb was recorded using Optotrak (Northern Digital), an optoelectronic tracking device that recorded the positions of a $5 \times 5 \mathrm{~mm}$ IRED attached to the tip of the index finger. Infrared emitting diode (IRED) positions were sampled at $200 \mathrm{~Hz}$. EMG activity of seven shoulder and elbow muscles was recorded using surface electrodes (Delsys). Electrodes consisted of three $1 \times 10 \mathrm{~mm}$ parallel silver bars placed $10 \mathrm{~mm}$ apart and were housed in a compact case containing a $10 \times$ preamplifier. Electrodes were placed to record the activity of the pectoralis clavicular head (shoulder flexor), biceps brachii short head (bi-articular flexor acting at the shoulder and elbow), biceps brachii long head (bi-articular flexor acting primarily at the elbow), posterior deltoid (shoulder extensor), triceps brachii long head (biarticular extensor acting at the shoulder and elbow), and triceps brachii lateral head (elbow extensor). For nine subjects, we recorded from brachioradialis (elbow flexor) instead of biceps long head. Electrode placement was verified using a number of test maneuvers including movement and isometric force tasks (Gribble and Ostry 1998, 1999; Gribble et al. 2002). EMG signals were amplified 1,000 times, analog band-pass filtered between 20 and $450 \mathrm{~Hz}$, and digitally sampled at $1,200 \mathrm{~Hz}$. Data collection and target presentation were controlled using custom software programmed in LabView (National Instruments).

\section{Data analysis}

Position signals were digitally low-pass filtered at $15 \mathrm{~Hz}$ using a second-order Butterworth filter implemented in Matlab (Mathworks). EMG signals were full-wave rectified and then low-pass filtered at 50 Hz. Baseline noise was subtracted from EMG signals based on signal levels recorded when the subject was relaxed and pointing to the central target. For each trial, signals were time-aligned to movement onset, which was scored by identifying the time at which tangential velocity of the fingertip exceeded $50 \mathrm{~mm} / \mathrm{s}$. Movement end was scored by identifying the time at which tangential velocity fell below 50 $\mathrm{mm} / \mathrm{s}$ and stayed below this threshold for $\geq 200 \mathrm{~ms}$. 
Movement accuracy was assessed using two measures of endpoint error: constant error and variable error. Constant error was defined as the mean distance between the fingertip at movement end and each target location. Variable error was defined as the mean distance between the endpoint of each trial and the overall average endpoint position within each condition (Darling and Gilchrist 1991; Rossetti et al. 1994). Thus constant error gives a measure of the overall accuracy with respect to target position, and variable error provides a measure of endpoint variability. We also examined accuracy during movement using two measures: first by computing trajectory error (with respect to a straight line) and second by computing the variability of hand trajectories. To compute trajectory error, the distance between each point along the hand trajectory and a straight line connecting start and end targets was computed and then averaged over the entire movement. To compute trajectory variability, each hand trajectory was first time-normalized to 300 samples, and the SD of the $x$ and $y$ components of trajectories was computed and averaged over time for the 20 movements to each target size and location.

Measures of cocontraction were estimated for each trial both during movement and immediately following movement end by considering EMG activity of antagonist muscles at the shoulder (posterior deltoid and pectoralis), elbow (triceps lateral head and biceps long head/ brachioradialis), and biarticular muscles that span both joints (triceps long head and biceps short head). To facilitate comparisons between electrodes and across subjects, EMG values for each muscle were first normalized for each subject by dividing by the maximum observed EMG activity for that muscle over the course of the dataset. Thus normalized EMG values are expressed as a proportion of maximum observed phasic EMG activity. To verify that the results reported below were not due to the particular normalization procedure used, we repeated the analyses described here by normalizing EMG levels using z-scores (Gribble and Ostry 1998; Suzuki et al. 2001). The results were qualitatively similar to those reported below.

In the past, we have restricted the analysis of cocontraction to a short window of time following movement end (Gribble and Ostry 1998; Suzuki et al. 2001). Here we also estimated cocontraction by examining tonic EMG levels at movement end, by averaging normalized EMG activity within a 200-ms window. As a control for the possibility that EMG activity during the measurement window was influenced by phasic activity (e.g., tremor or terminal oscillations), we excluded from analyses those trials in which significant negative correlations $(P<0.01)$ were observed between EMG activity in opposing flexor and extensor muscle groups during the 200-ms measurement window. This resulted in the elimination of between 5 and $15 \%$ of trials across subjects.

Recently a measure of cocontraction during movement termed "wasted contraction" has been reported (Thoroughman and Shadmehr 1999), which is computed here in the following way. For a given agonist-antagonist muscle pair (e.g., pectoralis and posterior deltoid), at each sampling point in time the minimum value of (normalized) EMG is computed. This is equivalent to discarding the portion of EMG in one muscle that is not matched by EMG in the opposing muscle. The resulting time-varying signal represents the magnitude of normalized EMG that is equal and opposite in antagonist musclesEMG in opposing muscles that increases joint stiffness. Although this measure is subject to many simplifications (see DISCUSSION), it nevertheless provides an estimate of changes in the magnitude of muscle coactivation during movement. In this paper, we computed measures of cocontraction during movement for shoulder, elbow, and biarticular muscle pairs. The resulting time-varying signals were averaged between movement start and end to give overall measures of cocontraction during movement for each muscle group. In all cases repeated measures ANOVA and Tukey posthoc tests were used to test the statistical reliability of differences between means.

\section{R E S ULT S}

\section{Cocontraction}

Figure 2 shows mean time-varying muscle activation patterns for one subject, for the 30-mm target. EMG and hand tangential velocity signals are time-aligned to movement onset. For all four movement directions (Fig. 2, $A-D$ ), phasic bursts in agonist and antagonist muscles are seen, which are followed by a period of sustained tonic activity. Across subjects, levels of tonic EMG during the 200-ms measurement window after movement end ranged between 5 and $20 \%$ of the maximum observed phasic EMG for each muscle during movement.

A two-factor repeated measures ANOVA was used to test for differences in mean normalized tonic EMG following movement as a function of target size and direction. Significant main effects of target size were seen in all muscles $(P<0.05)$. Figure $3 A$ shows mean normalized tonic EMG as a function of target size for all muscles; tonic EMG increases as target size
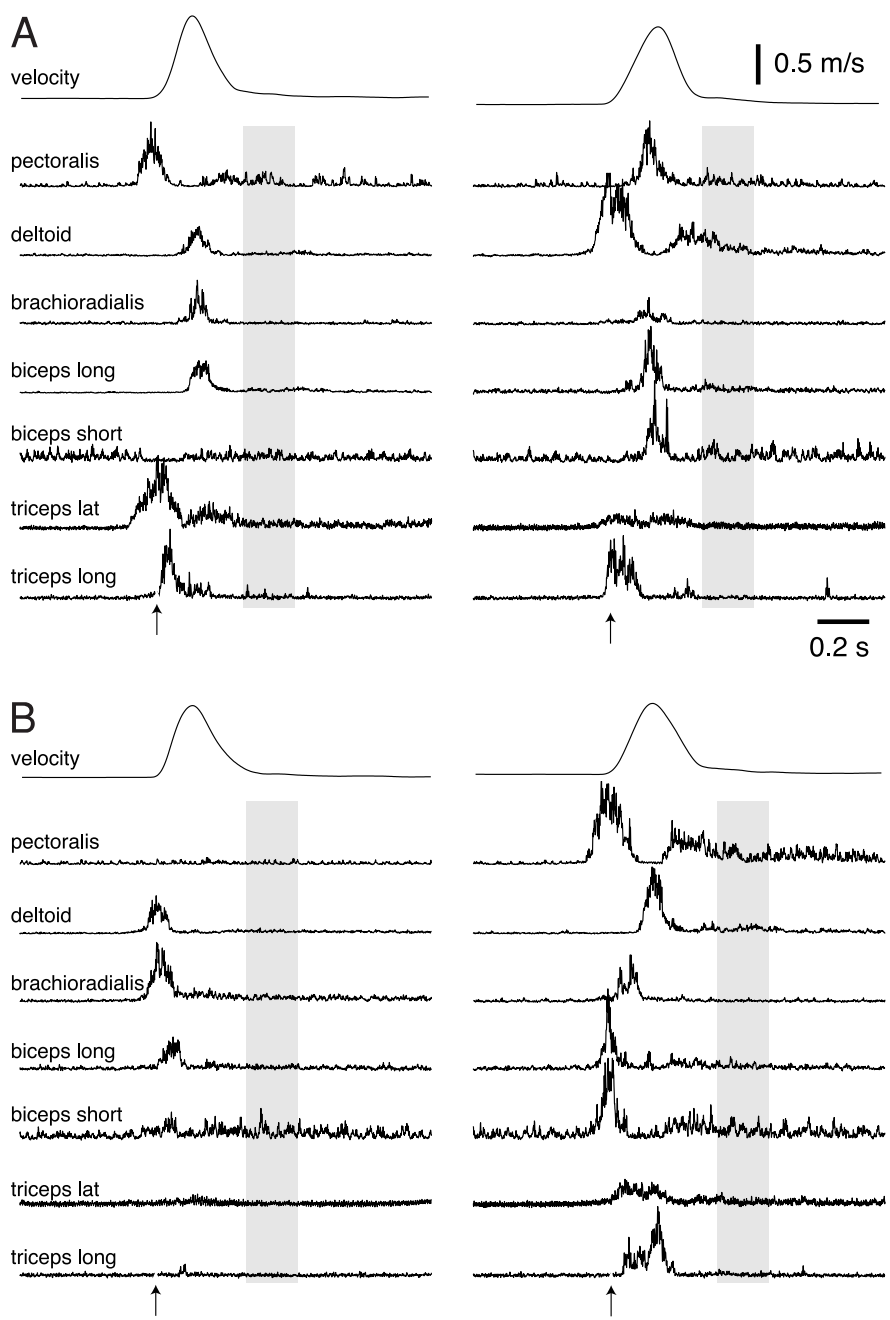

FIG. 2. Typical EMG records. Mean time-varying EMG activity from 7 shoulder, elbow, and biarticular muscles, and tangential velocity of the hand, is shown time-aligned to movement onset (indicated by vertical arrows), for movements to targets $1(A), 2(B) 3(C)$, and $4(D)$. Shaded areas represent the 200-ms window used to estimate tonic EMG after movement end (note that the figure presents mean EMG traces, but for the purposes of the analyses presented here, tonic EMG was estimated on a trial-by-trial basis). 


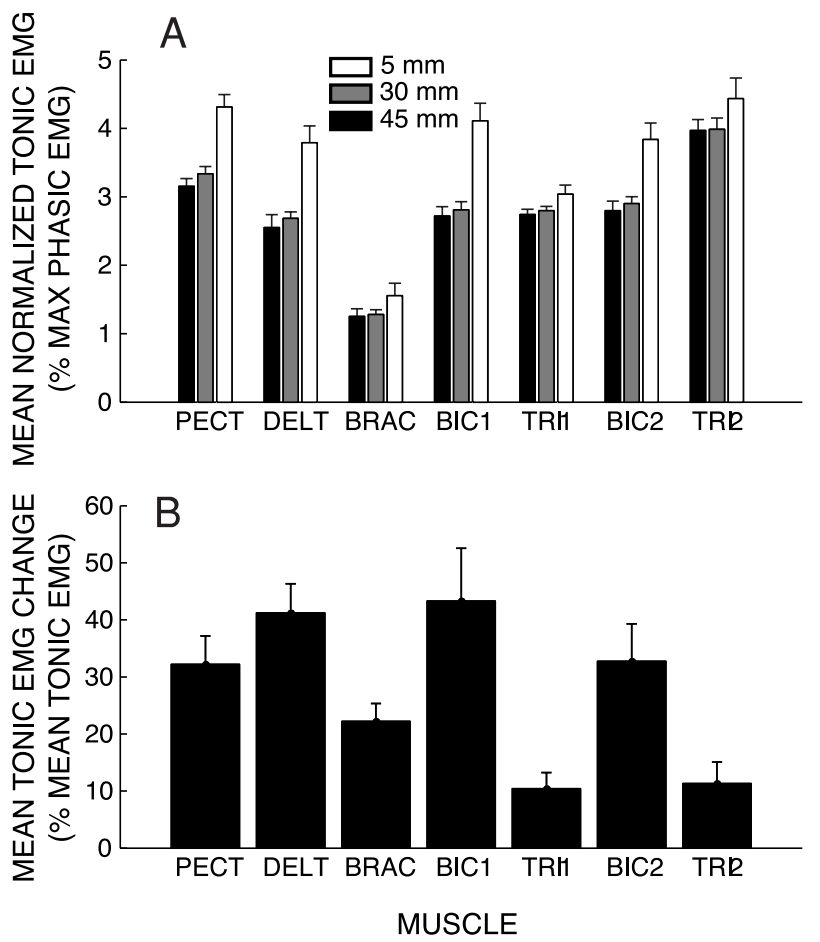

FIG. 3. Effect of target size on cocontraction at movement end. A: mean normalized tonic EMG plotted as a function of target size for 7 shoulder and elbow muscles. PECT: pectoralis clavicular head; DELT: posterior deltoid; BRAC: brachioradialis; BIC1: biceps long head; TRI1: triceps lateral head; BIC2: biceps short head; TRI2: triceps long head. $B$ : mean change in tonic EMG from the 45- to 5-mm targets plotted as a proportion of overall mean tonic EMG. Vertical bars represent SE.

decreases. Posthoc tests showed significant differences between tonic EMG levels for the 5- and 45-mm targets and between the 5 -and $30-\mathrm{mm}$ targets $(P<0.05$ for all muscles). Figure $3 B$ shows the mean change in tonic EMG between the 5- and 45-mm targets, expressed as a proportion of the mean tonic EMG across all target sizes and locations. Tonic EMG changes ranged between 10 and 50\% of mean tonic EMG. A significant main effect of target direction on tonic EMG levels was also seen $(P<0.05$ for all muscles). This effect of target position on tonic EMG at movement end presumably reflects differences in the levels of afferent contributions to motorneuron activation due to changes in limb posture (Sergio and Kalaska 1997), and/or differences in voluntary activation necessary to hold the limb at the final position against internal forces of stretched elastic tissue.

Significant effects of target size were also seen on cocontraction during movement. Figure $4 A$ shows mean cocontraction during movement as a function of target size for shoulder, elbow, and biarticular muscle pairs. In all cases, there was a significant linear trend for cocontraction to increase as target size decreases $(P<0.05)$. For all muscles, posthoc tests show significant differences between cocontraction levels for the 45versus $30-\mathrm{mm}$ targets $(P<0.05)$ and the 30 - versus $5-\mathrm{mm}$ targets $(P<0.01)$, with the exception of elbow muscles, for which no significant differences were observed between the $45-$ and $30-\mathrm{mm}$ targets $(P>0.05)$. Figure $4 B$ shows mean change in cocontraction between the 5- and $45-\mathrm{mm}$ targets, expressed as a proportion of the mean cocontraction across all target sizes. Changes in cocontraction during movement ranged from 5 to $15 \%$ of the mean and were largest for shoulder muscles.

We directly assessed the relationship between cocontraction and movement accuracy on a trial-by-trial basis. Figure 5 shows mean normalized tonic EMG after movement end plotted as a function of endpoint accuracy (constant error). Data shown represent mean values for 16 subjects averaged across the four target locations. For the purposes of visualization, data for brachioradialis and biceps long head have been combined. In all cases, a significant negative correlation was observed $(P<0.01$ for all muscles except triceps long head, $P<$ 0.05 ) - more accurate movements were associated with higher levels of tonic EMG at movement end. The same pattern was observed for cocontraction during movement, which is shown in Fig. 6. Movements that resulted in more accurate endpoint location were associated with higher levels of cocontraction during movement in shoulder, elbow, and biarticular muscles $(P<0.01$ in all cases $)$.

We assessed the correspondence between measures of cocontraction taken at the end of movement (tonic EMG) and during movement. On a trial-by-trial basis, significant correlations were observed in all subjects between cocontraction during and after movement. Figure 7 shows mean cocontraction during movement plotted as a function of mean tonic EMG at movement end, averaged over all subjects for each of the 12 experimental conditions (4 target locations $\times 3$ sizes). Significant correlations between the two cocontraction measures
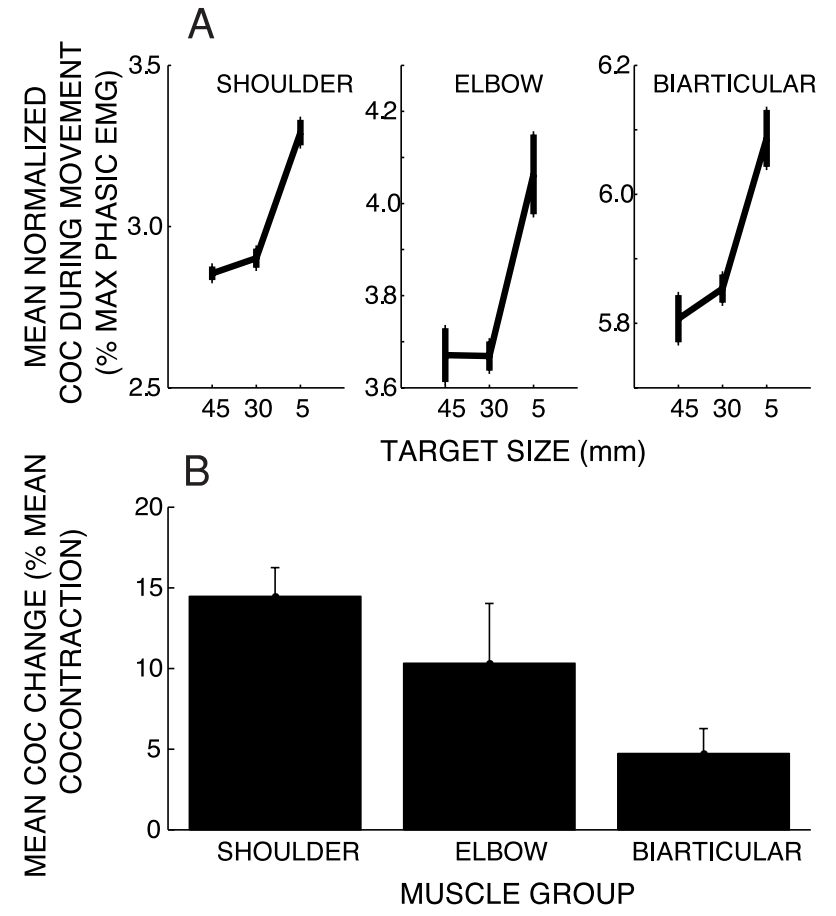

FIG. 4. Effect of target size on cocontraction during movement. A: mean normalized cocontraction during movement plotted as a function of target size for 3 muscle groups: shoulder (pectoralis and posterior deltoid), elbow (biceps long head or brachioradialis and triceps lateral head), and biarticular (biceps short head and triceps long head). $B$ : mean change in cocontraction during movement from the 45- to 5-mm targets plotted as a function of overall mean cocontraction. Vertical bars indicate SE. 


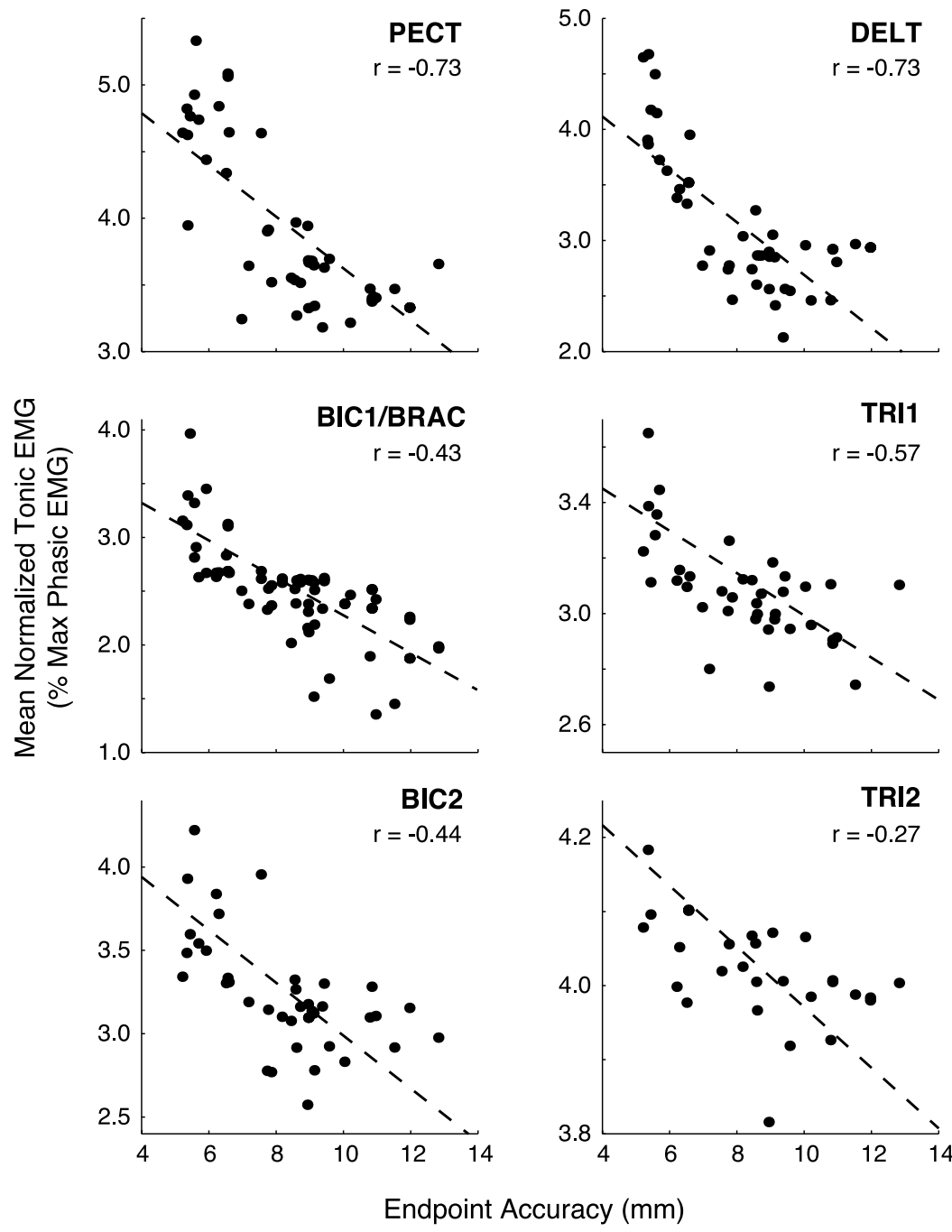

FIG. 5. Direct relationship between cocontraction after movement end and movement accuracy. Mean normalized tonic EMG activity after movement end is plotted as a function of endpoint accuracy. Data for 16 subjects are shown for 3 target sizes, averaged over the 4 target locations. For the purposes of visualization, data for brachioradialis and biceps long head have been combined. were observed for shoulder $(r=0.946, P<0.01)$, elbow $(r=$ $0.825, P<0.01)$, and biarticular $(r=0.837, P<0.01)$ muscle groups.

\section{Movement accuracy}

As target size decreased, movement accuracy improved, both during and at the end of movement. Accuracy during movement was assessed using two measures: trajectory error and trajectory variability (see METHODS). A significant decrease in both trajectory error and variability was observed as target size decreased $(P<0.05$; see Fig. 8). For both measures, posthoc tests show significant decreases between the 45- and 30-mm targets $(P<0.05)$ and 30 - and 5-mm targets $(P<$ $0.01)$.

We also examined changes in endpoint accuracy; Fig. 9 shows endpoint error plotted as a function of target size. There was a significant linear trend for both constant and variable error measures to decrease as target size decreased $(P<0.05)$. Posthoc tests show significant decreases in constant error between the 45 - and $30-\mathrm{mm}$ targets $(P<0.05)$ and 30 - and $5-\mathrm{mm}$ targets $(P<0.01)$, and significant decreases in variable error for 45 - versus 5-mm targets $(P<0.05)$ and 30-versus 5-mm targets $(P<0.01)$. No statistically significant decrease in variable error was observed for the 45-versus $30-\mathrm{mm}$ targets $(P>0.05)$. Endpoint error effectively decreased by one-half from the 45 - to 5-mm target.

\section{Kinematic controls}

To rule out the possibility that the observed changes in EMG could be due to changes in kinematic properties of movement that may have covaried with target size, we examined a number of kinematic variables. No significant differences were observed in average velocity $[P>0.05,0.320 \pm 0.021(\mathrm{SD}) \mathrm{m} / \mathrm{s}]$ or movement duration $(P>0.05,468 \pm 39 \mathrm{~ms})$ as a function of target size. To rule out the possibility that the observed changes in tonic EMG may have been influenced by phasic activity during the measurement window, we excluded trials in which significant negative correlations were observed between opposing muscles (see METHODS). In addition, we tested for a possible relationship between target size and hand movement during the 200-ms tonic EMG measurement window at movement end. No significant differences were observed for hand movement distance $(P>0.05,2.1 \pm 0.11 \mathrm{~mm})$ or mean tangential velocity $(P>0.05,0.025 \pm 0.001 \mathrm{~m} / \mathrm{s})$ as a function of target size. 


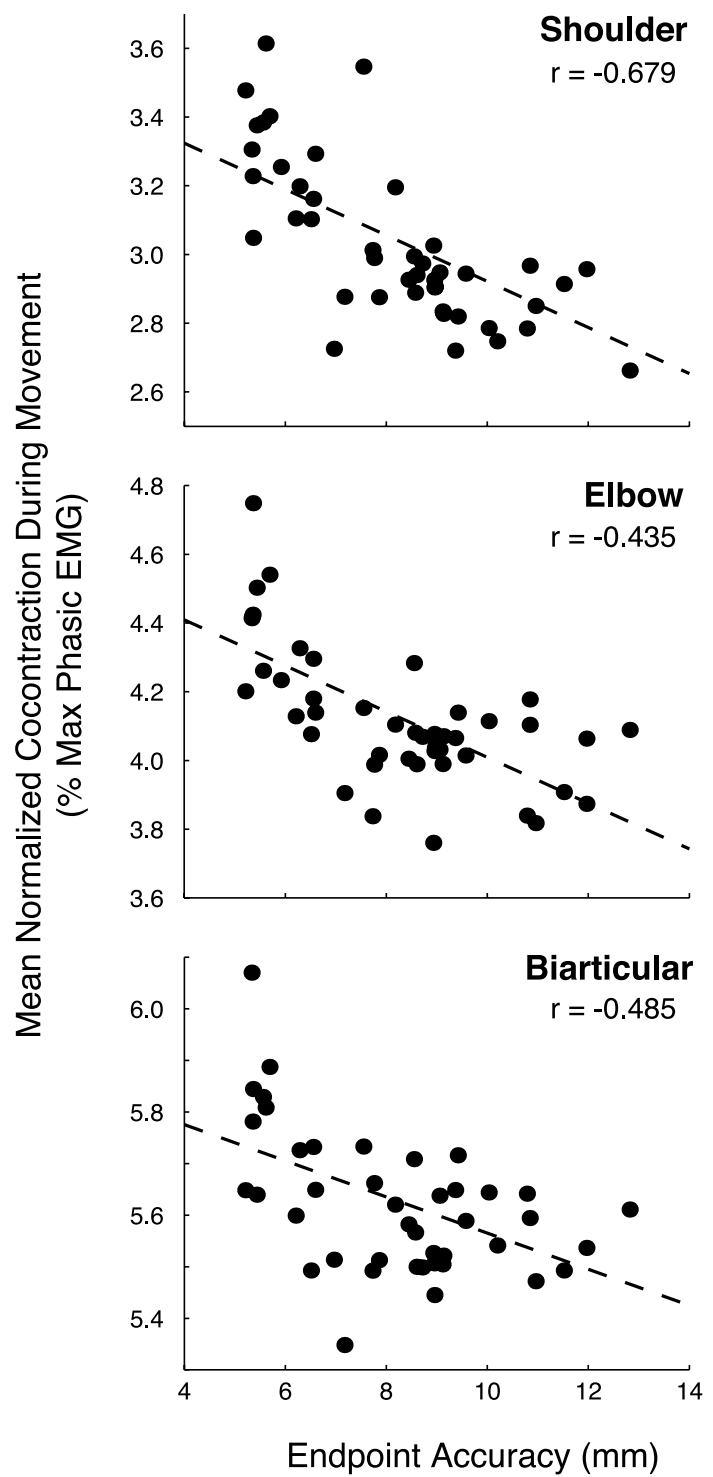

FIG. 6. Direct relationship between cocontraction during movement and accuracy. Mean normalized cocontraction during movement is plotted as a function of endpoint accuracy. Data for 16 subjects are shown averaged over 4 target locations.

\section{Practice effects}

To assess possible effects of practice on cocontraction, we grouped trials into six consecutive blocks of 40 trials. Figure 10 shows mean normalized tonic EMG and mean normalized cocontraction during movement as a function of movement block for shoulder, elbow, and biarticular muscle pairs. A relatively small, but statistically significant, decrease in both tonic EMG and cocontraction during movement was observed for all three muscle pairs $(P<0.05$ in all cases). The average decrease in normalized cocontraction over the course of the experiment ranged across subjects from 0.5 to $2.0 \%$ of maximum phasic EMG. While these decreases may appear small, when expressed relative to overall mean normalized cocontraction levels, they represent changes of 10 to $50 \%$.

To rule out the possibility that the observed decreases in cocontraction levels may have been caused by nonspecific decreases in the magnitude of recorded EMG signals (for example, due to changes in the electrode-skin interface over time), we tested for changes in the magnitude of peak phasic EMG bursts over the course of the experiment. No statistically reliable changes in the magnitude of phasic EMG bursts were observed over the 6 blocks ( $P>0.05$ for all muscles).

\section{I S C U S S I O N}

We examined EMG activity of shoulder and elbow muscles to assess a potential relationship between changes in muscle cocontraction and movement accuracy. Cocontraction both during movement and during a $200-\mathrm{ms}$ window beginning at movement end varied as a function of target size. As target size decreased, cocontraction in shoulder, elbow, and biarticular muscles increased, and measures of movement error decreased. This suggests that the CNS may use changes in cocontraction as a way to facilitate movement accuracy. Increases in muscle coactivation and corresponding increases in joint impedance provide greater limb stability and may represent a strategy to minimize the perturbing effects of forces arising from limb dynamics (Gribble and Ostry 1998; Milner 2002) and potential perturbing forces from external loads (Gomi and Haggard

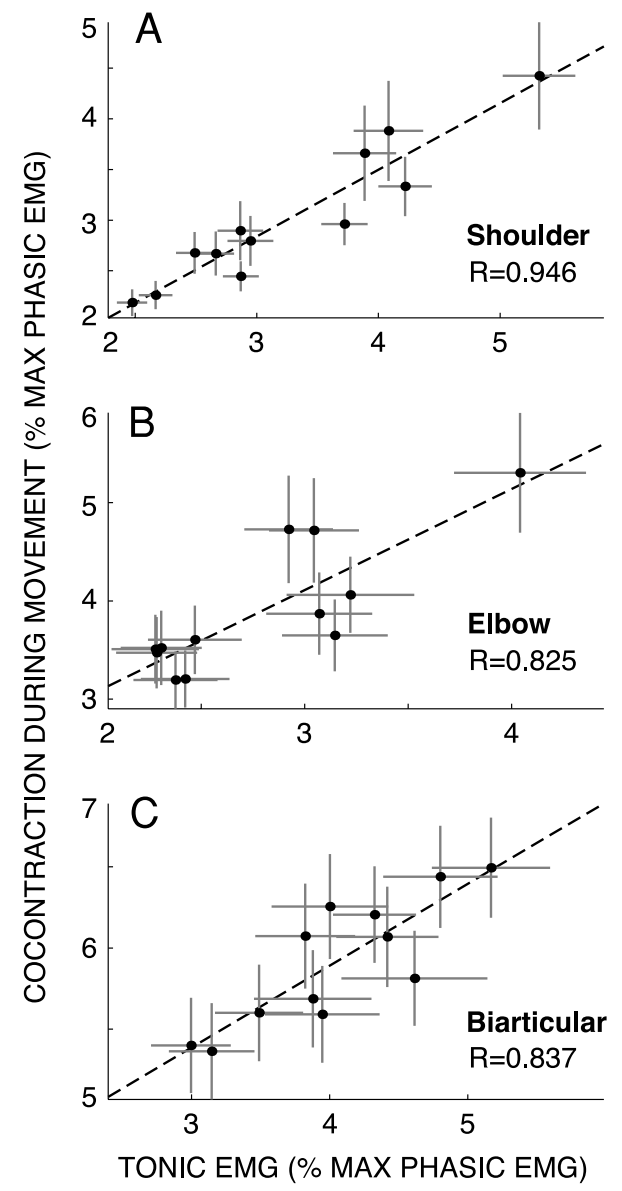

FIG. 7. Correspondence between cocontraction at movement end and during movement. Mean normalized cocontraction during movement is plotted as a function of mean normalized tonic EMG at movement end for each of 12 experimental conditions ( 4 target locations $\times 3$ target sizes). Significant correlations between cocontraction during and after movement were observed for shoulder $(A)$, elbow $(B)$, and biarticular $(C)$ muscle pairs. Mean normalized tonic EMG values for flexor and extensor muscle pairs were averaged to provide elbow, shoulder, and biarticular values. Horizontal and vertical bars represent SE. 

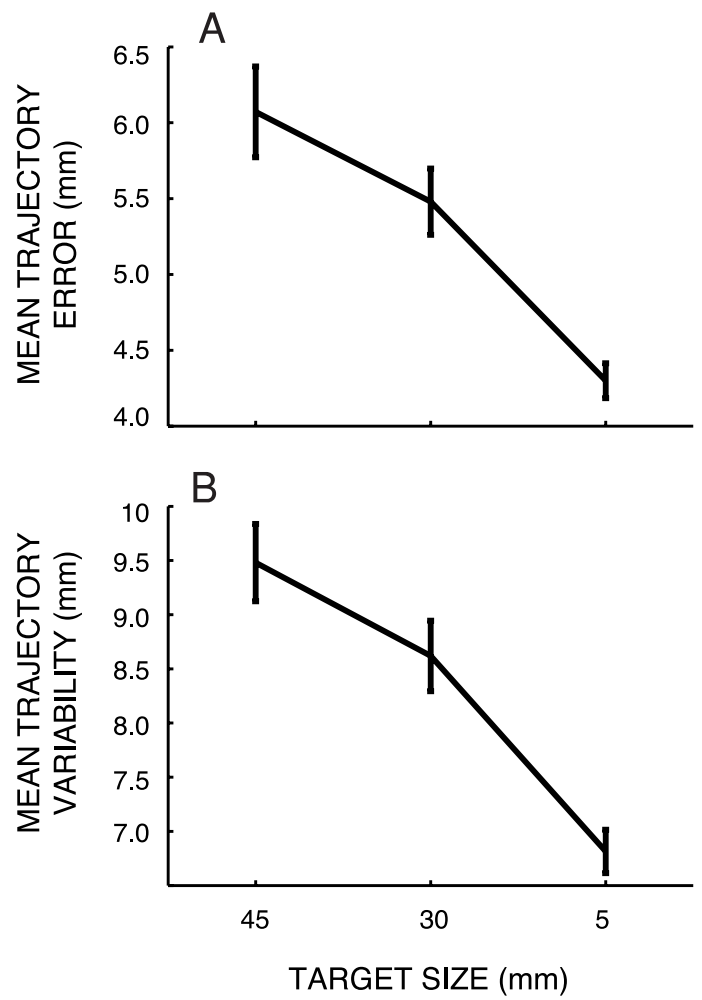

FIG. 8. Effect of target size on trajectory error and variability. A: mean error between movement trajectories and a straight line connecting start and end targets is plotted as a function of target size. $B$ : mean variability of hand trajectories (see METHODS) is plotted as a function of target size.

2001; Lacquaniti and Maioli 1989; Milner and Cloutier 1998; Thoroughman and Shadmehr 1999). The observations here that trajectory variability decreased and movements became straighter supports the notion that cocontraction was used to provide stability to the moving limb.

Behavioral studies of single-joint postural tasks have shown that subjects use muscle coactivation as a strategy to stabilize limb joints in the presence of external loads (De Serres and Milner 1991; Kearney and Hunter 1990; Milner and Cloutier 1998). More recently, it has been demonstrated that subjects are able to independently modulate the relative balance of cocontraction and limb stiffness in different spatial directions (Burdet et al. 2001; Gomi and Haggard 2001) and at different joints (Gribble and Ostry 1998). Cocontraction of antagonist muscles has also been observed during movement in which torque must be generated very quickly; Gordon and Ghez $(1984,1987)$ showed that cocontraction can be used to cancel a portion of agonist torque, so as not to exceed a desired target level. Similar situations arise when torque direction must be controlled at a joint that has multiple degrees of freedomcocontraction of an antagonist muscle may be used to cancel torque components orthogonal to the desired direction. In this study, no tendency was observed for target positions to be consistently overshot; thus presumably the changes in cocontraction observed here are not an attempt by the CNS to reduce antagonist torque but rather an attempt to increase the mechanical stability of the moving limb. This is supported by the observation that trajectory error and variability decreased as cocontraction increased.

Given the growing body of evidence that the motor system

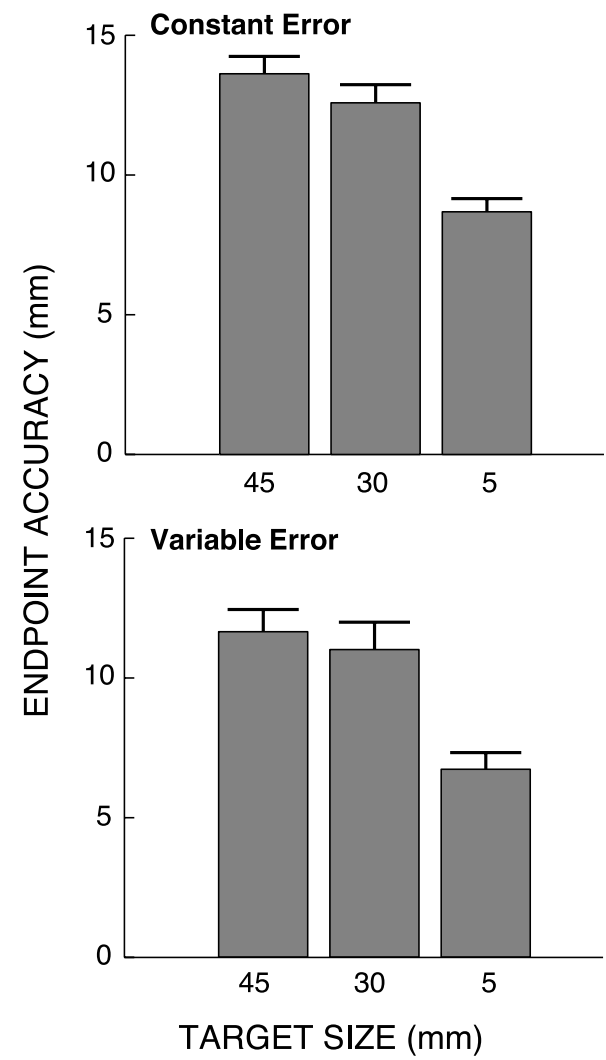

FIG. 9. Effect of target size on endpoint accuracy. Constant and variable error are plotted as a function of target size, averaged over the 4 target locations. Both measures of movement error decreased significantly as target size was reduced. Vertical bars indicate SE.

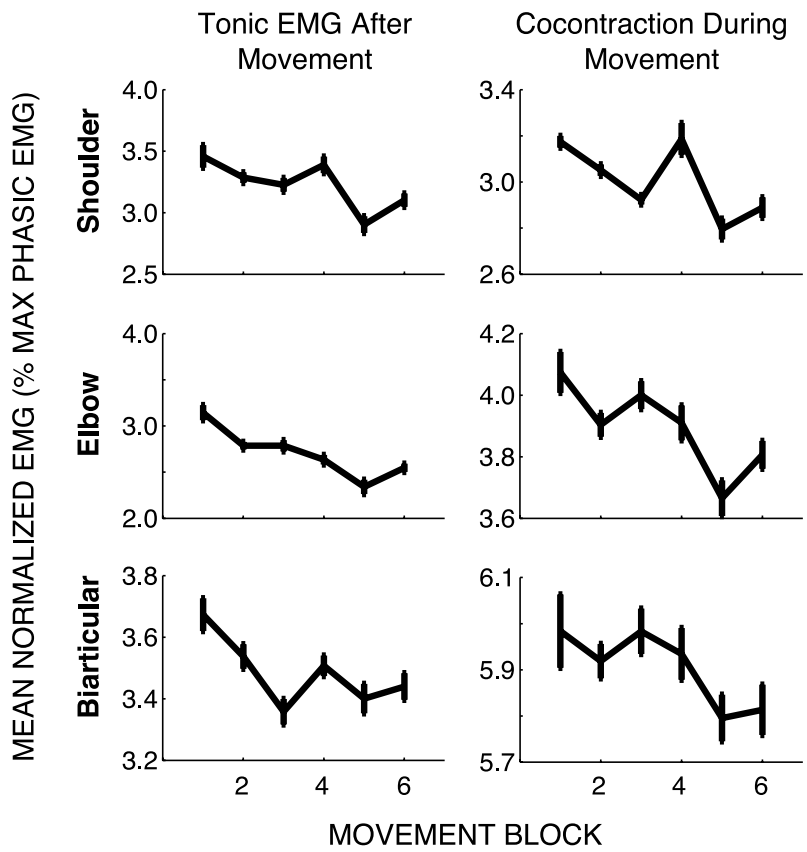

FIG. 10. Effect of practice on cocontraction. Mean normalized tonic EMG at movement end (left) and cocontraction during movement (right) are plotted as a function of movement block for shoulder, elbow, and biarticular muscle pairs. In all cases, a significant decrease in cocontraction was observed over the course of the experiment. Vertical bars denote $1 \mathrm{SE}$. 
is capable of forming feedforward, "internal models" of mechanical and dynamical contexts including joint interaction torques (Gribble and Ostry 1999; Koshland et al. 2000; Sainburg et al. 1995, 1999), one may raise the question of why the CNS used cocontraction and not changes in reciprocal muscle activation to achieve greater movement accuracy. It has been proposed that cocontraction may be a strategy that is used by the CNS early in learning a novel motor task to achieve greater accuracy in the absence of a fully formed "internal model" of dynamics - and that with ongoing practice, cocontraction may be reduced as learning takes place and internal representations are built up to achieve greater accuracy using changes in reciprocal, feedforward motor commands (Osu et al. 2002). In support of this idea, we found evidence that the magnitude of cocontraction, both during movement and at movement end, was gradually reduced over the course of the experiment. This is consistent with recent demonstrations that limb stiffness and EMG activity in antagonist muscles are modulated over the course of learning novel arm movement tasks (Osu et al. 2002; Thoroughman and Shadmehr 1999). The hypotheses that the CNS regulates cocontraction and hence limb stiffness as a way to facilitate movement accuracy, and that cocontraction is gradually decreased with practice, are both consistent with the results of the present study.

Previous studies of the control of cocontraction have used measures of tonic EMG following movement end to estimate cocontraction levels (Gribble et al. 1998; Suzuki et al. 2001). A concern has been that it is difficult to separate time-varying EMG signals into portions due to central changes in cocontraction, commands for movement, and afferent contributions to motorneuron activation. Here we estimated cocontraction both by examining tonic EMG levels after movement and with the use of a recently reported measure of antagonist coactivation termed "wasted contraction" (Thoroughman and Shadmehr 1999). The rationale behind the measure is to restrict the analysis to those portions of (normalized) EMG activity that are equal and opposite in flexor and extensor muscle pairs. Such activity, regardless of its specific origin, is presumed to nevertheless contribute to an increase in joint impedance. The pattern of results in the present paper were highly consistent using both measures, and indeed, high correlations were observed between tonic EMG levels at movement end and the measures of cocontraction computed during movement. This supports the idea that changes in the level of cocontraction are specified not only at movement end, to maintain posture, but also during movement, to provide stability to the moving limb.

In this study, we used the mean wasted contraction levels between movement start and end as an index of overall cocontraction during movement. Although beyond the scope of the present investigation, it would be useful in future studies to examine in more detail the degree to which cocontraction levels vary during different phases of movement and how the relative balance of stiffness from shoulder, elbow, and biarticular muscles may change over the course of movement. Nonmonotonic patterns of time-varying limb stiffness have been reported for multi-joint arm movements (Gomi and Kawato 1996, 1997). Stiffness at the shoulder and elbow and crosscoupling stiffness terms were initially low, and then increased at the beginning of movement, decreased near peak velocity, increased again during the deceleration phase, and finally decreased again at movement end. However, it has since been shown using physiologically based computational modeling studies that these nonmonotonic patterns of stiffness during movement are predicted by a model that combines a constantrate shift in the equilibrium position of the limb with an independent cocontraction command that is raised to a constant level throughout movement (Gribble et al. 1998). Further studies, in particular those in which EMG measures of cocontraction are combined with direct measurements of limb stiffness will aid in determining the relationship between muscle cocontraction, limb stiffness, and their potential modulation over the course of multi-joint movements.

While useful as a first approximation, the measure of cocontraction during movement used here has a number of limitations. The calculation of time-varying cocontraction is based only on surface EMG signals and thus does not take into account factors such as differences in muscle moment arms, differences in muscle force-generating ability, or possible varying contributions to cocontraction from other muscles not monitored in this study. In addition, while the relationship between muscle force and measured EMG has been well established in statics (Hunter and Kearney 1982; Osu and Gomi 1999), this relationship has not been fully assessed during movement. Presumably, muscle mechanical properties such as the force-velocity relation affect the relationship between instantaneous muscle force and the magnitude of surface EMG signals during motion of the limb. Nevertheless, as a first approximation, the measure used here is useful as a rough estimate of how opposing agonist/antagonist activity during movement changes with movement parameters such as target size. In addition, the high degree of correspondence between the measure of cocontraction during movement and tonic EMG levels measured after movement end, when the limb was stationary, provides a degree of confidence that the observed changes in EMG during movement are indeed related to muscle coactivation.

Several theories have proposed that various dynamic variables may be minimized by the CNS for movement planning and control, including the minimum torque change and minimum commanded torque change models (Klein Breteler et al. 2002; Nakano et al. 1999; Uno et al. 1989). In the present study, cocontraction levels were seen to increase as target size and movement error decreased. Although this may be a mechanically effective way to stabilize the limb, muscle coactivation is metabolically expensive, and thus with respect to energetic considerations alone, would not represent an optimal strategy for movement control. However, it has been suggested that in the presence of noise (e.g., fluctuations in muscle force), the optimal compromise between energy consumption and postural positioning error does in fact require antagonist muscle coactivation (Hogan 1984). Thus the changes in cocontraction observed here may represent a solution to the problem of balancing positional accuracy against energetic constraints in the face of signal-dependent noise.

Measures of EMG activation in antagonist muscles were used here to estimate levels of centrally specified cocontraction. These changes in muscle coactivation are presumably associated with corresponding changes in limb impedance (Kearney and Hunter 1990; Milner and Cloutier 1998; Osu et al. 2002). Although beyond the scope of this study, it would be of great interest in future work to relate the changes in antagonist muscle activation seen here to direct measures of limb 
impedance - both during and at the end of movement. Devices that are capable of perturbing the limb in different directions and measuring restoring forces have been used in the past to characterize the impedance of the limb (Burdet et al. 2001; Flash and Mussa-Ivaldi 1990; Gomi and Haggard 2001; Shadmehr et al. 1993; Tsuji et al. 1995) and other articulators such as the mandible (Shiller et al. 2002).

It is likely that the air-sleds used in this study to support the arm against gravity and reduce friction introduced destabilizing forces of a somewhat unpredictable nature. Presumably, these forces were relatively small, given that subjects were able to maintain limb positions both at the starting position and at each final target to within a few millimeters (see RESULTS) without extremely high levels of cocontraction. In addition, destabilizing forces introduced by the air-sleds presumably did not change systematically with target size. Nevertheless some component of the cocontraction observed here may be related to stabilization of the limb in the face of unpredictable airflow from the sleds. It is also possible that the arm was less stable than during many normal activities of daily living, given that shoulder muscles usually activated to hold the arm against gravity were not required, given the support provided by the airsleds.

In summary, we have shown that the changes in cocontraction at opposing shoulder, elbow, and biarticular muscles varies with movement accuracy. Knowledge about the behavioral determinants of cocontraction and limb impedance control is crucial to gain an understanding of how neural control signals for cocontraction are coordinated with those for multi-joint movement (Gribble and Ostry 1998; Gribble et al. 1998; Osu et al. 2002; Suzuki et al. 2001). This knowledge may also be used to aid in the design of neurophysiological studies aimed at understanding the neural bases of cocontraction and limb impedance control. In addition, knowledge about how the CNS specifies cocontraction levels in response to movement requirements may be used to further develop computational models of motor control that include control signals related to muscle cocontraction and limb stiffness (Feldman and Levin 1995; Flash 1987; Gribble and Ostry 2000; Gribble et al. 1998; Hogan 1985).

The authors thank D. Debicki and S. Everling for helpful comments.

This research was supported by grants from National Sciences and Engineering Research Council (Canada), Canadian Institutes of Health Research (Canada), the Canada Foundation for Innovation, and the Ontario Innovation Trust.

\section{REFERENCES}

Bhushan $\mathbf{N}$ and Shadmehr R. Computational nature of human adaptive control during learning of reaching movements in force fields. Biol Cybern 81: 39-60, 1999.

Burdet E, Osu R, Franklin DW, Milner TE, and Kawato M. The central nervous system stabilizes unstable dynamics by learning optimal impedance. Nature 414: 446-449, 2001.

Darling WG and Gilchrist L. Is there a preferred coordinate system for perception of hand orientation in three-dimensional space? Exp Brain Res 85: 405-416, 1991.

De Serres SJ and Milner TE. Wrist muscle activation patterns and stiffness associated with stable and unstable mechanical loads. Exp Brain Res 86: 451-458, 1991.

Feldman AG and Levin MF. The origin and use of positional frames of reference in motor control. Behav Brain Sci 18: 723-806, 1995.
Fitts PM. The information capacity of the human motor system in controlling the amplitude of movement. J Exp Psychol 47: 381-391, 1954.

Fitts PM and Peterson JR. Information capacity of discrete motor responses. J Exp Psychol 67: 103-112, 1964.

Flash T. The control of hand equilibrium trajectories in multi-joint arm movements. Biol Cybern 57: 257-274, 1987.

Flash T and Mussa-Ivaldi F. Human arm stiffness characteristics during the maintenance of posture. Exp Brain Res 82: 315-326, 1990.

Ghez C and Gordon J. Trajectory control in targeted force impulses. I. Role of opposing muscles. Exp Brain Res 67: 225-240, 1987.

Gomi $\mathbf{H}$ and Haggard P. Anticipatory stiffness regulation during arm movements. Soc Neural Cont Move Abstr 6: A1, 2001.

Gomi $\mathbf{H}$ and Kawato M. Equilibrium-point control hypothesis examined by measured arm stiffness during multijoint movement. Science 272: 117-120, 1996.

Gomi $\mathbf{H}$ and Kawato M. Human arm stiffness and equilibrium-point trajectory during multi-joint movement. Biol Cybern 76: 163-171, 1997.

Gordon J and Ghez C. EMG patterns in antagonist muscles during isometric contraction in man: relations to response dynamics. Exp Brain Res 55: 167-171, 1984.

Gribble PL, Everling S, Ford K, and Mattar A. Hand-eye coordination for rapid pointing movements. Arm movement direction and distance are specified prior to saccade onset. Exp Brain Res 145: 372-382, 2002.

Gribble PL and Ostry DJ. Compensation for interaction torques during single- and multijoint limb movement. J Neurophysiol 82: 2310-2326, 1999.

Gribble PL and Ostry DJ. Compensation for loads during arm movements using equilibrium-point control. Exp Brain Res 135: 474-482, 2000.

Gribble PL and Ostry DJ. Independent coactivation of shoulder and elbow muscles. Exp Brain Res 123: 355-360, 1998.

Gribble PL, Ostry DJ, Sanguineti V, and Laboissière R. Are complex control signals required for human arm movement? J Neurophysiol 79: 1409-1424, 1998.

Hogan N. Adaptive control of mechanical impedance by coactivation of antagonist muscles. IEEE Trans Auto Cont 29: 681-690, 1984.

Hogan N. The mechanics of multi-joint posture and movement control. Biol Cybern 52: 315-331, 1985.

Hunter IW and Kearney RE. Dynamics of human ankle stiffness: variation with mean ankle torque. J Biomech 15: 747-752, 1982.

Kearney RE and Hunter IW. System identification of human joint dynamics. Crit Rev Biomed Eng 18: 55-87, 1990.

Klein Breteler MD, Meulenbroek RG, and Gielen SC. An evaluation of the minimum-jerk and minimum torque-change principles at the path, trajectory, and movement-cost levels. Motor Control 6: 69-83, 2002.

Koshland GF, Galloway JC, and Nevoret-Bell CJ. Control of the wrist in three-joint arm movements to multiple directions in the horizontal plane. J Neurophysiol 83: 3188-3195, 2000.

Lacquaniti F and Maioli C. The role of preparation in tuning anticipatory and reflex responses during catching. J Neurosci 9: 134-148, 1989.

Latash ML. Independent control of joint stiffness in the framework of the equilibrium-point hypothesis. Biol Cybern 67: 377-384, 1992.

Milner TE. Adaptation to destabilizing dynamics by means of muscle cocontraction. Exp Brain Res 143: 406-416, 2002.

Milner TE and Cloutier C. Damping of the wrist joint during voluntary movement. Exp Brain Res 122: 309-317, 1998.

Nakano E, Imamizu H, Osu R, Uno Y, Gomi H, Yoshioka T, and Kawato M. Quantitative examinations of internal representations for arm trajectory planning: minimum commanded torque change model. J Neurophysiol 81: 2140-2155, 1999.

Osu R, Franklin DW, Kato H, Gomi H, Domen K, Yoshioka T, and Kawato M. Short- and long-term changes in joint co-contraction associated with motor learning as revealed from surface EMG. J Neurophysiol 88: 991-1004, 2002.

Osu R and Gomi H. Multijoint muscle regulation mechanisms examined by measured human arm stiffness and EMG signals. J Neurophysiol 81: 14581468, 1999.

Rossetti Y, Meckler C, and Prablanc C. Is there an optimal arm posture? Deterioration of finger localization precision and comfort sensation in extreme arm-joint postures. Exp Brain Res 99: 131-136, 1994.

Sainburg RL, Ghez C, and Kalakanis D. Intersegmental dynamics are controlled by sequential anticipatory, error correction, and postural mechanisms. J Neurophysiol 81: 1045-1056, 1999. 
Sainburg RL, Ghilardi MF, Poizner H, and Ghez C. Control of limb dynamics in normal subjects and patients without proprioception. J Neurophysiol 73: 820-835, 1995 .

Sergio LE and Kalaska JF. Systematic changes in directional tuning of motor cortex cell activity with hand location in the workspace during generation of static isometric forces in constant spatial directions. J Neurophysiol 78: 1170-1174, 1997.

Shadmehr R, Mussa-Ivaldi FA, and Bizzi E. Postural force fields of the human arm and their role in generating multijoint movements. $J$ Neurosci 13: 45-62, 1993.

Shiller DM, Laboissière R, and Ostry DJ. Relationship between jaw stiffness and kinematic variability in speech. J Neurophysiol 88: 2329-2340, 2002.

Soechting JF. Effect of target size on spatial and temporal characteristics of a pointing movement in man. Exp Brain Res 54: 121-132, 1984.
Suzuki M, Shiller DM, Gribble PL, and Ostry DJ. Relationship between cocontraction, movement kinematics and phasic muscle activity in singlejoint arm movement. Exp Brain Res 140: 171-181, 2001.

Thoroughman KA and Shadmehr R. Electromyographic correlates of learning an internal model of reaching movements. J Neurosci 19: 8573-8588, 1999.

Todorov E. Direct cortical control of muscle activation in voluntary arm movements: a model. Nat Neurosci 3: 391-398, 2000.

Tsuji T, Morasso PG, Goto K, and Ito K. Human hand impedance characteristics during maintained posture. Biol Cybern 72: 475-485, 1995.

Uno Y, Kawato M, and Suzuki R. Formation and control of optimal trajectory in human multijoint arm movement. Minimum torque-change model. Biol Cybern 61: 89-101, 1989. 